\title{
Fed-batch SSCF using steam-exploded wheat straw at high dry matter consistencies and a xylose-fermenting Saccharomyces cerevisiae strain: effect of laccase supplementation
}

Antonio D Moreno ${ }^{1,2+}$, Elia Tomás-Pejó ${ }^{2 \dagger}$, David Ibarra ${ }^{3}$, Mercedes Ballesteros ${ }^{4}$ and Lisbeth Olsson ${ }^{2 *}$

\begin{abstract}
Background: Lignocellulosic bioethanol is expected to play an important role in fossil fuel replacement in the short term. Process integration, improvements in water economy, and increased ethanol titers are key considerations for cost-effective large-scale production. The use of whole steam-pretreated slurries under high dry matter (DM) conditions and conversion of all fermentable sugars offer promising alternatives to achieve these goals.

Results: Wheat straw slurry obtained from steam explosion showed high concentrations of degradation compounds, hindering the fermentation performance of the evolved xylose-recombinant Saccharomyces cerevisiae KE6-12 strain. Fermentability tests using the liquid fraction showed a higher number of colony-forming units (CFU) and higher xylose consumption rates when treating the medium with laccase. During batch simultaneous saccharification and co-fermentation (SSCF) processes, cell growth was totally inhibited at $12 \% \mathrm{DM}(\mathrm{w} / \mathrm{v})$ in untreated slurries. However, under these conditions laccase treatment prior to addition of yeast reduced the total phenolic content of the slurry and enabled the fermentation. During this process, an ethanol concentration of $19 \mathrm{~g} / \mathrm{L}$ was obtained, corresponding to an ethanol yield of 39\% of the theoretical yield. By changing the operation from batch mode to fed-batch mode, the concentration of inhibitors at the start of the process was reduced and 8 $\mathrm{g} / \mathrm{L}$ of ethanol were obtained in untreated slurries with a final consistency of $16 \% \mathrm{DM}(\mathrm{w} / \mathrm{v})$. When fed-batch SSCF medium was supplemented with laccase 33 hours after yeast inoculation, no effect on ethanol yield or cell viability was found compared to untreated fermentations. However, if the laccase supplementation (21 hours after yeast inoculation) took place before the first addition of substrate (at 25 hours), improved cell viability and an increased ethanol titer of up to $32 \mathrm{~g} / \mathrm{L}$ ( $51 \%$ of the theoretical) were found.
\end{abstract}

Conclusions: Laccase treatment in SSCF processes reduces the inhibitory effect that degradation compounds have on the fermenting microorganism. Furthermore, in combination with fed-batch operational mode, laccase supplementation allows the fermentation of wheat straw slurry at high DM consistencies, improving final ethanol concentrations and yields.

Keywords: Lignocellulose, Bioethanol, Simultaneous saccharification and co-fermentation, In situ laccase detoxification, Steam explosion, Xylose-fermenting Saccharomyces cerevisiae

\footnotetext{
* Correspondence: lisbeth.olsson@chalmers.se

${ }^{\dagger}$ Equal contributors

${ }^{2}$ Department of Chemical and Biological Engineering, Industrial Biotechnology,

Chalmers University of Technology, Göteborg SE-41296, Sweden

Full list of author information is available at the end of the article
} 


\section{Background}

In terms of volume and market value, biomass is considered to be the most important source of renewable energy and bioethanol is considered to be the main alternative for fossil fuel replacement in the transportation sector in the short to medium term [1].

Lignocellulosic biomass is an abundant, low-cost, and widely distributed feedstock that can be used as raw material for the production of biofuels and other value-added products in future biorefineries. Biochemical conversion of lignocellulose to ethanol is, however, hindered by the complex and recalcitrant structure of these materials. To increase biomass digestibility, several pretreatment methods have been developed, with hydrothermal technologies such as steam explosion being one of the most commonly used [2,3]. During steam explosion, the accessibility of enzymes to cellulose is increased due to the solubilization of hemicelluloses and the redistribution of lignin. Inevitably, different by-products (weak acids, furan derivatives, and phenolic compounds) that inhibit enzymes and fermentation microorganisms in the subsequent steps are also generated in the process [4-7]. The inhibitory levels partly depend on fermentation variables including the physiological conditions of the cell, the dissolved oxygen tension, and the $\mathrm{pH}$ of the medium. Thus, by adjusting the fermentation conditions the inhibitory effects can be alleviated.

There are different ways to overcome the effects of the inhibitory compounds. One of the most commonly used strategies to avoid inhibition is to remove the inhibitors from the slurry using physical, chemical, or biological detoxification methods. Of the different biological detoxification strategies, in situ laccase treatment has been considered a more feasible option than other methods because such treatment does not require extra equipment and it is performed under mild reaction conditions [8]. Laccase enzymes have been purified from different ligninolytic microorganisms, mainly white-rot fungi, and they can oxidize substituted phenols, anilines, and aromatic thiols by reducing oxygen to water [9]. A wide range of pretreated materials have been subjected to laccase detoxification, resulting in improved fermentability after the treatment [10-14].

Agricultural residues such as wheat straw have been shown to be promising feedstocks for future biorefineries. Wheat straw is a very suitable material for bioethanol production because it is composed of high amounts of glucose and xylose, the latter being of utmost importance when xylose-fermenting yeasts are available. Saccharomyces cerevisiae KE6-12 (modified with XYL1 and XYL2 genes encoding xylose reductase (XR) and xylitol dehydrogenase $(\mathrm{XDH})$ from Pichia stipitis, respectively), which can consume both glucose and xylose from lignocellulosic materials in the presence of degradation compounds, represents a good choice as this strain has been shown to consume xylose completely with a $90 \%$ theoretical ethanol yield even on a demonstration scale $\left(10 \mathrm{~m}^{3}\right)$ [15].

There is no doubt about the need to increase the substrate loading in the fermentation process to reach higher ethanol concentrations and make the process economically viable. Even so, the use of whole slurries at high substrate loading will mean higher amounts of degradation products, in which case the use of strains that are highly inhibitor tolerant would be crucial. In the present study, the authors combined the use of the evolved xyloserecombinant $S$. cerevisiae KE6-12 strain with laccase enzyme treatment, with the purpose of increasing the final ethanol concentration using whole wheat straw slurry. Batch and fed-batch SSCF processes were run under high dry matter (DM) conditions to compare the ethanol concentrations, sugar consumption, and cell viability in untreated and laccase-treated fermentations.

\section{Results and discussion}

\section{Steam-explosion pretreatment}

Pretreated material was characterized and its composition is shown in Table 1. After steam explosion, the collected wheat straw slurry had a total DM content of $26 \%(\mathrm{w} / \mathrm{v})$, $21.5 \%(\mathrm{w} / \mathrm{v})$ of which was water-insoluble solids (WIS). The WIS fraction of the slurry was mainly composed of

\section{Table 1 Composition of pretreated wheat straw}

\begin{tabular}{|c|c|}
\hline Compound & Pretreated material $\left(210^{\circ} \mathrm{C}, 2.5 \text { minutes }\right)^{\mathrm{a}}$ \\
\hline \multicolumn{2}{|l|}{ WIS $(\%(W / W))$} \\
\hline Cellulose & 47.4 \\
\hline Hemicellulose & 8.4 \\
\hline Lignin & 25.4 \\
\hline Others & 1.5 \\
\hline \multicolumn{2}{|c|}{ Liquid fraction $(\%(w / w))$} \\
\hline Glucan & 1.0 \\
\hline Glucose & 0.3 \\
\hline Xylan & 6.6 \\
\hline Xylose & 0.7 \\
\hline Arabinan & 0.1 \\
\hline Arabinose & 0.4 \\
\hline Galactan & 0.4 \\
\hline Galactose & 0.2 \\
\hline Total phenol & 2.6 \\
\hline Acetic acid & 1.6 \\
\hline Formic acid & 2.1 \\
\hline Furfural & 0.19 \\
\hline 5-HMF & 0.07 \\
\hline
\end{tabular}

${ }^{a}$ Values expressed as g/100 g of slurry (dry weight). 5-HMF, 5hydroxymethylfurfural; WIS, water-insoluble solids. 
cellulose $(47.4 \% \mathrm{w} / \mathrm{w})$ and lignin $(25.4 \% \mathrm{w} / \mathrm{w})$, with minor hemicellulose content $(8.4 \% \mathrm{w} / \mathrm{w})$ (Table 1$)$.

As a consequence of hemicellulose solubilization, a high xylose concentration (mostly in the oligomeric form) was measured and different degradation products were identified and quantified as soluble compounds (Table 1). These compounds are considered to be inhibitors and can affect biochemical pathways in the fermenting microorganisms and interact with cellulolytic enzymes, leading to reduced final ethanol titers and volumetric productivities $[6,7,16,17]$. Acetic acid, formic acid, and phenolic compounds were the most abundant degradation products in terms of percentage $(\mathrm{w} / \mathrm{w})$ of total slurry (Table 1$)$. Acetic acid is derived from the acetyl groups present in hemicelluloses [18]. At low $\mathrm{pH}$ in the fermentation medium, the acetic acid $(\mathrm{pKa}=$ 4.7 ) is in the undissociated form, is liposoluble, and diffuses into the cells. Inside the cell $(\mathrm{pH}=7.4)$, the acid dissociates causing a decrease in $\mathrm{pH}$ that inhibits different activities and promotes an energy imbalance by removing these ions through ATP pumps [16]. Since the formation of acetic acid is inherent to hemicellulose hydrolysis, its formation cannot be prevented. Furfural and 5-hydroxymethylfurfural (5-HMF) were also identified in the slurry, and they are produced by pentose and hexose dehydration, respectively. These compounds affect cell growth and respiration rates, and most yeast used for ethanol production can reduce the aldehyde group on the furan ring to convert them into less toxic alcoholic forms [17]. The ability of yeasts to transform furfural and 5-HMF offers a way of in situ detoxification. To some extent, this encourages resistance to furans, or the yeasts may gradually become adapted to their presence. Further degradation of furfural and 5-HMF generates formic acid, which has similar inhibitory action to that of acetic acid. In addition to the inhibitors already mentioned, a variety of aromatic, polyaromatic, phenolic, and aldehydic compounds are released from the lignin fraction $[18,19]$. Among them, a wide variety of substituted phenols and cinnamic acids such as vanillin, syringaldehyde, 4-hydroxybenzaldehyde, ferulic acid, and $p$-coumaric acid have previously been reported to be present in steamexploded wheat straw [19-21]. These compounds can cause partitioning and loss of integrity of cell membranes, reducing both the specific growth rate and the assimilation of sugars. Phenols, especially low-molecular-weight compounds, have a considerable inhibitory effect and are more toxic than furfural and 5-HMF (even at low concentrations), and their effects are difficult to alleviate by adjusting the fermentation conditions $[17,18]$. In addition to this, phenolic compounds can also inhibit and deactivate hydrolytic enzymes [6,7].

\section{Effect of laccase on the liquid fraction fermentation} In order to evaluate the fermentability of the pretreated material and the ability of the evolved recombinant strain to ferment xylose in the presence of degradation compounds, the liquid fraction from pretreated slurry was diluted to concentrations corresponding to different DM content and fermented using different inoculum sizes $(1,3$, and $5 \mathrm{~g} / \mathrm{L})$. Moreover, to test the effects of laccase on the fermentability of the liquid fraction, $P y c$ noporus cinnabarinus laccase was added to the culture medium at $1 \mathrm{IU} / \mathrm{mL}$. The number of colony-forming units $(\mathrm{CFU} / \mathrm{mL})$ dropped rapidly in untreated liquid fractions equivalent to $14 \%$ DM content, independently of inoculum size, showing that the toxic compounds had a fatal effect on the fermenting microorganism (Figure 1). When laccase treatment was used, $73 \%$ of the phenol content was removed (the concentration decreased from 4.8 to $1.3 \mathrm{~g} / \mathrm{L}$ ) and an increase in the inoculum size from 1 to 3 or $5 \mathrm{~g} / \mathrm{L}$ resulted in maintenance of cell viability after 24 hours from inoculation. The concentration of weak acids or furan derivatives was not affected by the laccase treatment (data not shown), which has also been seen in other investigations $[10,14,21,22]$.

Detoxification by laccases implies an oxidative polymerization mechanism. Laccases extract one electron from phenols, generating unstable phenoxy radicals that can interact with each other, leading to polymerization into large-size aromatic compounds, which are less toxic for fermentative microorganisms [10]. During this process, the selective action of laccases on different phenols establishes a faster conversion for syringaldehyde or cinnamic acids, while vanillin is oxidized at lower rates and other compounds such as 4-hydroxybenzaldehyde are not affected $[20,22]$. Thus, non-laccase-active compounds or those that are oxidized at lower rates can remain in the medium and, together with weak acids and furan derivatives, have an inhibitory effect on fermentative strains, mainly reducing the volumetric ethanol productivity.

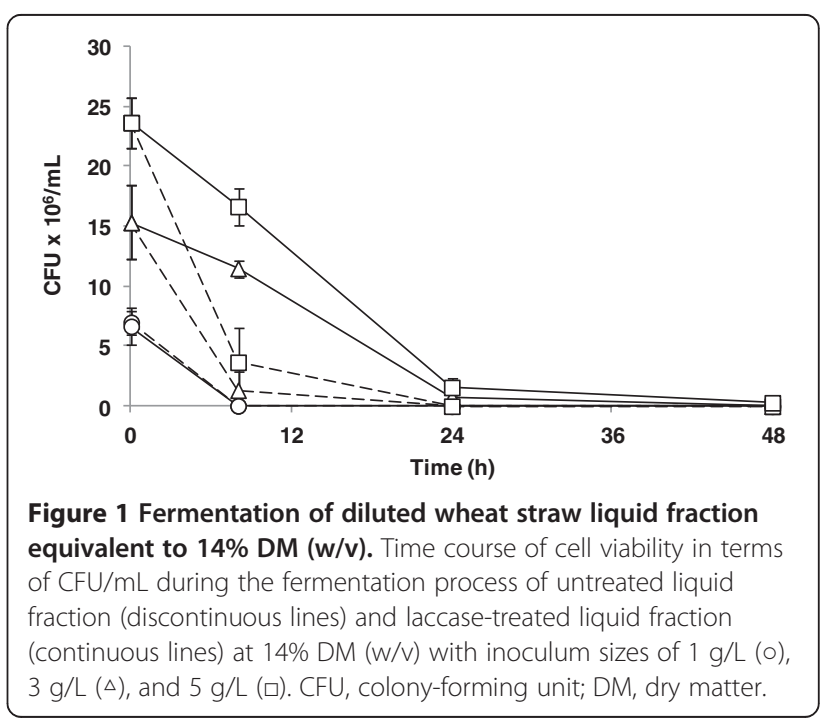


To improve cell viability and xylose consumption, the liquid fraction was diluted to the equivalent of $12 \% \mathrm{DM}$ content and inoculated with $5 \mathrm{~g} / \mathrm{L}$ of evolved $S$. cerevisiae KE6-12, because of the best growth at this inoculum size when using the liquid fraction corresponding to $14 \%$ DM. Due to the lower concentration of inhibitors in the fermentation medium at the equivalent to $12 \%$ $\mathrm{DM}$, the cell viability was maintained throughout the 120-hour long process, even when no laccase treatment was given (Figure 2A). Glucose was the first sugar to be depleted in both untreated and laccase-treated liquid fractions, and no differences in consumption rates were found (Figure 2B). Following glucose consumption, xylose was consumed at lower rates and a shorter lag phase for laccase-treated liquid fractions led to higher xylose consumption rates at the initial stage of fermentation. Nevertheless, analysis of variance (ANOVA) did not show statistically significant differences between the ethanol yields at the $95.0 \%$ confidence level. The reduction in the lag phase can be attributed to the $81 \%$ decrease in phenolic content by the action of laccase (Table 2). As explained above, phenolic compounds can alter biological membranes, thus affecting the growth of fermenting microorganisms [16]. Lower amounts of

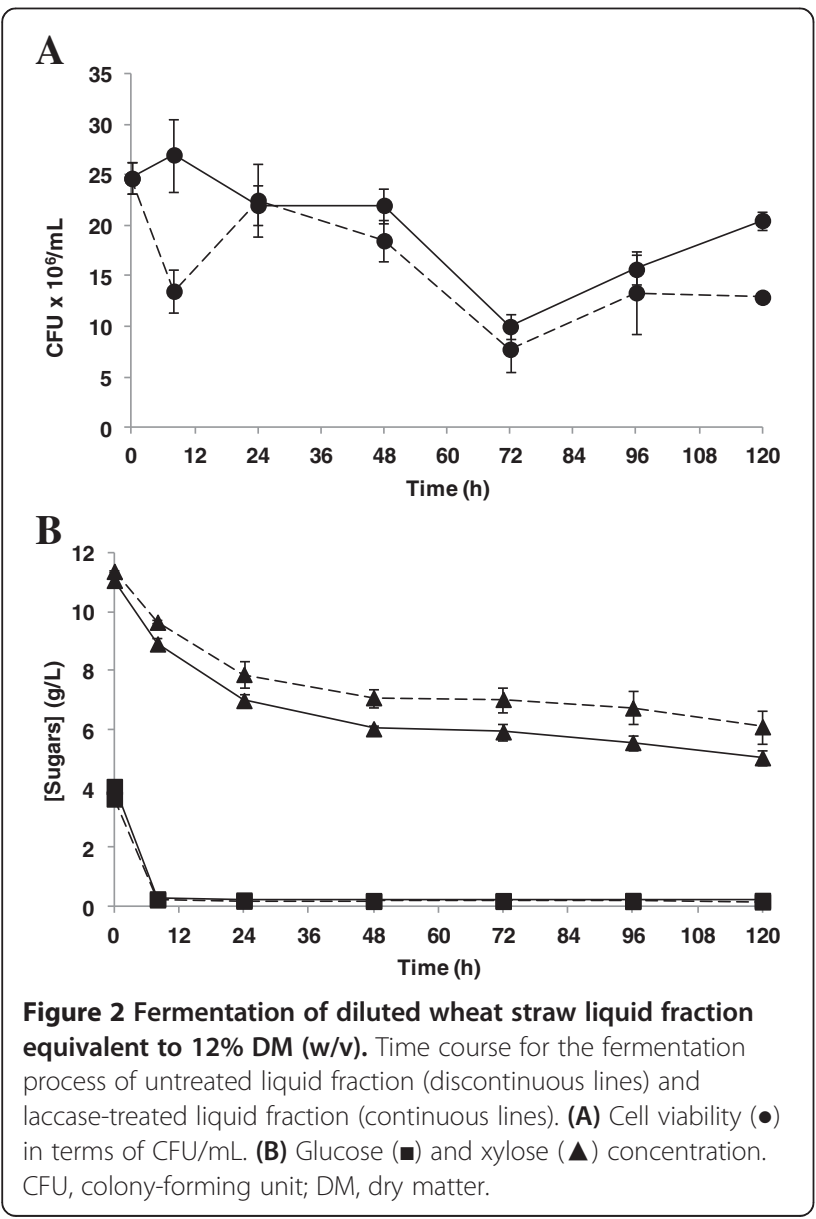

soluble phenols during fermentation favor cell growth and better ethanol yield [11-14].

\section{SSCF of wheat straw slurry in batch mode}

Taking into account the results obtained during the fermentation of the liquid fraction, $12 \% \mathrm{DM}(\mathrm{w} / \mathrm{v}) \mathrm{di}-$ luted slurries were subjected to SSCF processes in batch mode. In this case, the inoculum size of $S$. cerevisiae KE6-12 was reduced to $1 \mathrm{~g} / \mathrm{L}$ in order to minimize this parameter and enable detection of differences after laccase treatment. As illustrated in Figure 3A, cell viability was lost within the first 24 hours and neither sugar consumption nor ethanol concentration was observed in untreated slurries (Figure 3B,C). On the other hand, laccase treatment prior to the SSCF process reduced the amount of soluble phenols in slurries, allowing growth of the fermenting microorganism, and a maximum number of CFU $/ \mathrm{mL}$ was reached at 24 hours. Laccasetreated slurries had $77 \%$ less phenols than untreated ones. This value was slightly lower than the observed one for the liquid fraction, even when the treatment was 9 hours longer. This result could be explained by mixing problems when using diluted slurry [23]. Limitation in terms of mixing and mass transfer can affect the homogeneity of the slurry and diminishing the accessibility of phenols to the action of laccase $[20,24]$.

When the substrate consistency was increased from $12 \%$ to $16 \% \mathrm{DM}(\mathrm{w} / \mathrm{v})$, no cell growth was observed, even in laccase-treated slurries, due to the high concentration of inhibitory compounds and greater mass transfer limitations. The use of laccases for the detoxification of wheat straw slurry has already been described. Jurado et al. (2009) [12] found an increase in ethanol concentration of 2 to 2.7 times when enzymatic hydrolysates from both acid and non-acid steam-pretreated materials were detoxified with laccases from Coriolopsis rigida and Trametes villosa. In the same way, Moreno et al. (2012) [14] used laccases from $P$. cinnabarinus and $T$. villosa to detoxify the whole slurry from steam-exploded wheat straw, allowing the thermotolerant yeast Kluyveromyces marxianus CECT 10875 to ferment diluted slurries that could not be fermented before treatment. Furthermore, these authors have also reported an ethanol concentration of up to $22 \mathrm{~g} / \mathrm{L}$ when laccase-treated slurries were fermented with the xylose-fermenting $S$. cerevisiae F12 strain, even in the absence of an external nitrogen source [24].

Due to the lag phase shown by S. cerevisiae KE6-12 after inoculation in batch SSCF at $12 \%$ DM (w/v), glucose and xylose concentrations increased at the start of the process (Figure 3B). The presence of both glucose and xylose usually results in a delay in xylose consumption during co-fermentation, as these sugars share the transporter by which they are transported into the cells 
Table 2 Initial phenols, glucose, and xylose, and maximum ethanol concentration and ethanol yield

\begin{tabular}{ccccccc}
\hline Liquid fraction (equivalent \% $(w / v))$ & Phenols $_{\text {ini }}(\mathbf{g} / \mathbf{L})$ & $\mathrm{EtOH}_{\max }(\mathbf{g} / \mathbf{L})$ & Glucose $_{\text {ini }}(\mathbf{g} / \mathbf{L})$ & $\mathrm{Xylose}_{\text {ini }}(\mathbf{g} / \mathbf{L})$ & $\mathbf{Y}_{\mathrm{E} / \mathrm{s}}(\mathbf{g} / \mathbf{g})^{\mathbf{a}}$ \\
\hline \multirow{2}{*}{$12 \% \mathrm{DM}$} & Untreated & 4.2 & 3.7 & 3.7 & 11.4 & $0.25^{\mathrm{b}}$ \\
& Laccase & 0.8 & 4.2 & 4.1 & 11.1 & $0.28^{\mathrm{b}}$ \\
\hline
\end{tabular}

${ }^{\mathrm{a}}$ Ethanol yield based on total sugars measured prior to fermentation; ${ }^{\mathrm{b}}$ no statistically significant differences. Fermentation of liquid fraction with an inoculum size of $5 \mathrm{~g} / \mathrm{L}$. DM, dry matter; EtOH, ethanol; ini, initial; max, maximum.

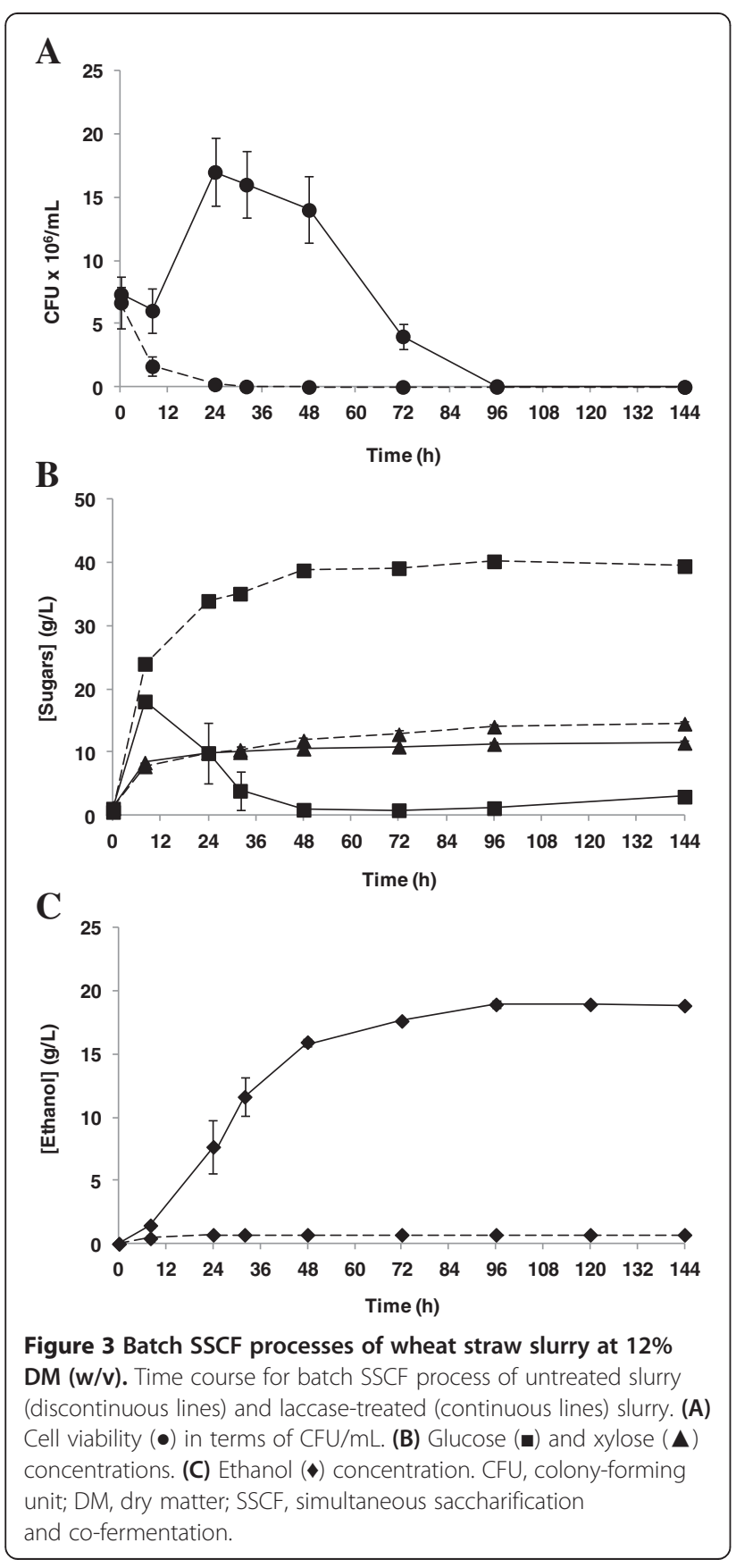

[25]. Thus, a preference for glucose over xylose can limit the consumption of xylose, therefore explaining its higher concentration at the end of the fermentation. A final ethanol concentration of $19 \mathrm{~g} / \mathrm{L}$ was obtained in laccase-treated slurries (Figure $3 \mathrm{C}$ ). This value corresponds to a final ethanol yield of $0.20 \mathrm{~g} / \mathrm{g}$ based on the total amount of glucose and xylose from the solid and liquid fractions of the diluted slurries (glucose from enzyme preparations was also taken into account). Assuming a theoretical ethanol yield of $0.51 \mathrm{~g} / \mathrm{g}$ for both sugars, 39\% of the maximum amount of possible ethanol resulted under these conditions. However, when only the sugars released during saccharification were considered (with values determined from untreated slurries), the final yield increased to $70 \%$, showing the enzymatic hydrolysis to be an important limiting factor. An efficient saccharification step is essential to obtain higher ethanol concentrations. Development of new enzymatic cocktails with improved activities can contribute to better hydrolytic performances, increasing the availability of sugar during fermentation $[26,27]$. In addition, the use of thermotolerant strains that can ferment at temperatures close to the optimal for saccharification could improve this step, thus contributing to increase final ethanol concentrations [19,28].

\section{SSCF of wheat straw slurry in fed-batch mode}

For cost-effective bioethanol production, ethanol concentrations above $4 \%(\mathrm{w} / \mathrm{v})$ are needed to reduce distillation costs [29]. By increasing substrate loadings, higher concentrations of fermentable sugars are available and then higher ethanol concentrations can be achieved [30]. Increasing the solids content, however, would also lead to higher concentrations of inhibitory compounds, which would explain why batch SSCF at 16\% DM (w/v) could not be fermented. One feasible alternative to achieve higher substrate loadings is to change the operational mode from batch to fed-batch. Under the latter configuration, the substrate is gradually added to the medium, keeping the concentration of inhibitors at levels suitable for fermentation, and in situ adaptation of microorganisms to inhibitory compounds could improve their tolerance towards a new substrate addition. Moreover, low initial substrate loadings avoid mixing problems and reduce accumulation of glucose, so a more efficient co-fermentation of xylose and glucose takes place [31,32]. 
The fed-batch assays were performed with an initial slurry content of $6 \% \mathrm{DM}(\mathrm{w} / \mathrm{v})$ and an inoculum size of $3 \mathrm{~g} / \mathrm{L}$ (but when considering all substrate additions, the inoculum size was $1.2 \mathrm{~g} / \mathrm{L}$ ). After 25 hours and 50 hours of fermentation, slurry was added, reaching a substrate consistency of $12 \%$ and $16 \% \mathrm{DM}(\mathrm{w} / \mathrm{v})$, respectively. When using this fed-batch strategy, ethanol at $8.3 \mathrm{~g} / \mathrm{L}$ was produced in untreated slurries (Figure $4 \mathrm{C}$ ). It is
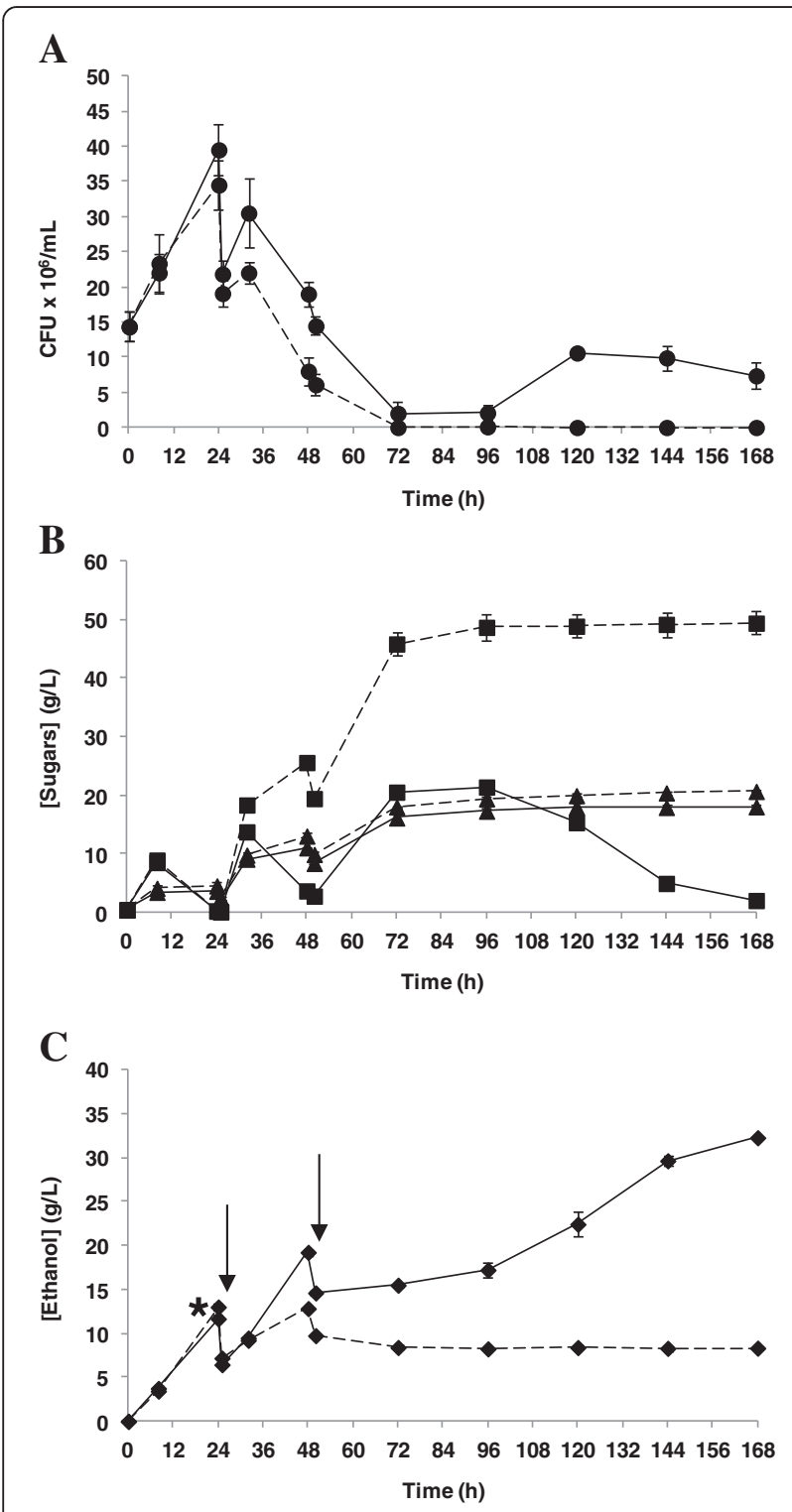

Figure 4 Fed-batch SSCF processes of wheat straw slurry at $16 \%$ DM (w/v). Time course for fed-batch SSCF process of untreated slurry (discontinuous lines) and laccase-treated slurry (continuous lines) (supplemented after 21 hours of inoculation). (A) Cell viability (•) in terms of CFU/mL. (B) Glucose (-) and xylose ( $\mathbf{\Delta}$ ) concentrations. (C) Ethanol ( concentration. The asterisk and arrows indicate addition of laccase and substrate, respectively. CFU, colony-forming unit; DM, dry matter; SSCF, simultaneous saccharification and co-fermentation. remarkable that no fermentation took place in batch mode at the same consistency. As mentioned above, in situ adaptation of the fermenting microorganism may take place during the fed-batch assay (Figure 4A,B). While fermentation at $12 \% \mathrm{DM}(\mathrm{w} / \mathrm{v})$ in batch mode was not possible for untreated slurries, a continuous increase in the ethanol concentration was observed when the same substrate consistency (after the first addition of substrate) was reached with the fed-batch strategy. However, after the second addition of substrate, cell growth ceased completely and the production of ethanol (and sugar consumption) suddenly stopped, resulting in accumulation of sugars due to the continued enzymatic action.

During fed-batch SSCF assays, laccase was added using different strategies. In one set of experiments, laccase was added 4 hours before the first addition of substrate and in a second set of experiments, it was added 8 hours after the first addition (at 21 hours and 33 hours of fermentation, respectively). In contrast to batch SSCF, a laccase treatment step prior to inoculation in fed-batch SSCF was not performed since the amounts of inhibitory compounds were low at the start of the process. Furthermore, it has been shown that laccase treatment prior to enzymatic hydrolysis reduces the saccharification yield of steam-exploded wheat straw $[12,14,24]$. Thus, a delay in addition of laccase can reduce this negative effect on enzymatic hydrolysis and minimize the effect of laccase treatment on saccharification yields. When laccase was added 21 hours after inoculation, the phenolic content during fermentation remained below $0.6 \mathrm{~g} / \mathrm{L}$ after detoxification (with total phenolic content measured at 32, 48 , and 72 hours after yeast inoculation). As a result, cell viability was enhanced, reaching the maximum CFU number at 24 hours and keeping the cells alive throughout the fermentation (Figure 4A). In terms of product concentration, $32.3 \mathrm{~g} / \mathrm{L}$ ethanol were found at the end of the process (Figure $4 \mathrm{C}$ ), corresponding to an ethanol yield of $0.26 \mathrm{~g} / \mathrm{g}$ ( $51 \%$ of theoretical). This yield was 1.3 times higher than that obtained with laccase-treated slurries at $12 \% \mathrm{DM}(\mathrm{w} / \mathrm{v})$ in batch mode, even when using higher substrate consistency (16\% DM (w/v)). An increase of the ethanol concentrations and yields when working with steam-exploded wheat straw in a fed-batch operation mode SSCF was also observed by other authors [19,31]. Tomás-Pejó et al. (2009) [19] reported an ethanol concentration of $36.2 \mathrm{~g} / \mathrm{L}$ and ethanol yield of $0.33 \mathrm{~g} / \mathrm{g}$ with a fed-batch strategy $(10 \% \mathrm{WIS}(\mathrm{w} / \mathrm{v})$ as initial substrate concentration that was increased until 14\% WIS (w/v) after 12 hours), compared with an ethanol concentration and yield of $30.2 \mathrm{~g} / \mathrm{L}$ and $0.27 \mathrm{~g} / \mathrm{g}$, respectively, in batch strategy at $14 \%$ WIS (w/v). In a similar way, Olofsson et al. (2008) [31] showed an increase from $59 \%$ in batch mode up to $71 \%$ in fed-batch mode, 
of the theoretical ethanol yield, using steam-pretreated wheat straw at a final WIS content of $9 \%$.

The higher ethanol yields in fed-batch SSCF can be explained due to the better saccharification yields during the fed-batch processes as in this operational mode the initial substrate consistencies are low. By increasing substrate consistency, a decrease in saccharification yields has previously been described due to end-product inhibition, unproductive binding, protein deactivation or denaturalization, and the decline in the binding capacity of enzymes to cellulose [33-35]. In this context, a lower initial substrate concentration could increase enzymatic hydrolysis yields, resulting in higher overall yields. Furthermore, since the inhibitors are kept at a lower level than in batch SSCF, higher co-consumption of glucose and xylose can be expected, considering also the fact that low glucose concentration at early stages of the process facilitates xylose consumption [15,31].

Either in untreated or laccase-treated slurry, an adaptation of the microorganism to the higher concentrations of inhibitors after each addition of substrate was required. This adaptation phase was more remarkable at critical stages (12\% DM (w/v) for untreated slurry and $16 \% \mathrm{DM}(\mathrm{w} / \mathrm{v})$ for laccase-treated slurry). During these adaptation phases, ethanol was produced at lower rates and glucose accumulated in the medium (Figure 4B,C).

In a second set of experiments, laccase was added after the first addition of substrate, at 33 hours after inoculation. Surprisingly, laccase supplementation resulted in the same ethanol concentration and cell viability profiles as those obtained with untreated slurries. Thus, after the second addition of substrate (50 hours), cell growth and ethanol concentration were not observed (data not shown), and glucose and xylose accumulated (Figure 5). Furthermore, a

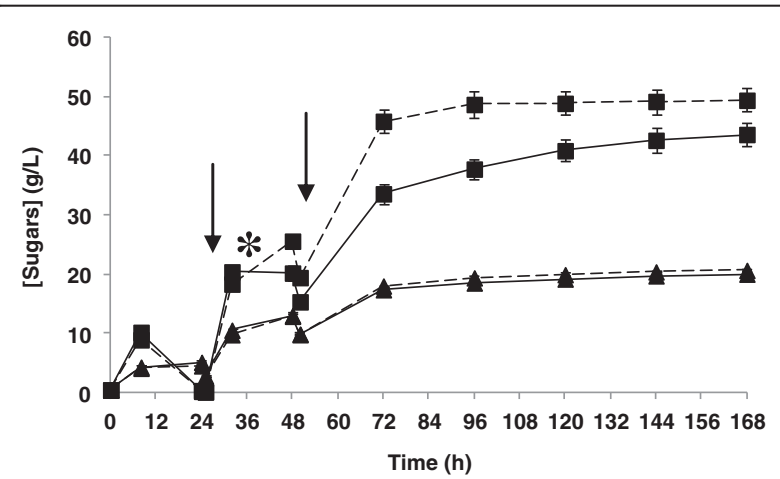

Figure 5 Profile of sugars during fed-batch SSCF process of wheat straw slurry at $16 \% \mathrm{DM}(\mathrm{w} / \mathrm{v})$. Saccharification profiles during a fed-batch SSCF process with untreated wheat straw slurry (discontinuous lines) and laccase-treated wheat straw slurry (continuous lines). Glucose ( $\mathbf{\square})$ and xylose ( $\mathbf{\Delta})$ concentrations. The asterisk and arrows indicate addition of laccase and substrate respectively. DM, dry matter; SSCF, simultaneous saccharification and co-fermentation. considerable difference in glucose concentration was observed between untreated and laccase-treated slurries. This result is in accordance with previous data indicating a clear effect in the saccharification step of steam-exploded wheat straw from the action of laccase [12,14]. Another interesting outcome from the fed-batch SSCF supplemented with laccase at 33 hours of fermentation was that no significant variations were found when comparing cell viability between untreated and laccase-treated slurries (data not shown), suggesting that the damage that resulted after exposure of cells to sublethal conditions could not be repaired immediately by reducing the concentration of degradation compounds. In addition, soluble phenols at concentrations of $1.2 \mathrm{~g} / \mathrm{L}$ and $1.7 \mathrm{~g} / \mathrm{L}$ were found after 48 hours and 72 hours, respectively. These phenolic values are higher than with the previous strategy (laccase addition at 21 hours) and could increase the synergistic effects of different inhibitors, hindering the fermenting microorganism from adapting to the culture medium.

\section{Conclusions}

The conversion of all fermentable sugars at high substrate loadings can contribute to making lignocellulosic bioethanol production economically viable. In the present work, laccase treatment was employed to overcome the effect of inhibitory compounds from steam-exploded wheat straw and to allow growth of the xylose-fermenting $S$. cerevisiae KE6-12. When wheat straw slurries were treated with laccase before fermentation, the phenolic content was considerably reduced and the slurries could be fermented at substrate consistencies of up to $12 \% \mathrm{DM}(\mathrm{w} / \mathrm{v})$. On the other hand, when using a fed-batch strategy the concentration of inhibitory compounds remains low at the start of the fermentation, allowing in situ adaptation of the fermenting microorganism to inhibitors and permitting the fermentation of slurries at $12 \% \mathrm{DM}$, even without laccase treatment. Moreover, the hydrolysis process is improved during fed-batch mode, resulting in higher saccharification yields and augmenting the concentrations of fermentable sugars. These benefits can be boosted by laccase supplementation, increasing the consistency of the slurry to $16 \%$ $\mathrm{DM}(\mathrm{w} / \mathrm{v})$, and thereby increasing the ethanol production to $32.3 \mathrm{~g} / \mathrm{L}$ and the overall yield up to $51 \%$. However, it is very important to optimize the timing of laccase treatment as this will determine the success of the process.

\section{Methods}

\section{Raw material and steam-explosion pretreatment}

Wheat straw to be used as the raw material was supplied by Ecocarburantes de Castilla y León (Salamanca, Spain) and had the following composition (\% dry weight): cellulose, 40.5; hemicellulose, 26.1; lignin, 18.1; and others, 15.3 [26].

The material was milled using a laboratory hammer mill in order to obtain small chips of 2 to $10 \mathrm{~mm}$, and 
these were stored at room temperature until used. Milled biomass was pretreated in a steam-explosion pilot plant carrying a $2 \mathrm{~L}$ reactor vessel. The temperature was fixed at $210^{\circ} \mathrm{C}$ and the residence time was fixed at 2.5 minutes. To obtain saturated steam at $210^{\circ} \mathrm{C}$, the pressure inside the vessel was maintained at 19 to 20 bars.

After pretreatment, one portion of collected slurry was vacuum-filtered to recover the liquid fraction and solids were thoroughly washed to obtain the WIS fraction.

\section{Enzymes}

P. cinnabarinus laccase (Beldem, Andenne, Belgium) was used for detoxification. The activity $(60 \mathrm{IU} / \mathrm{mL})$ was measured by oxidation of $5 \mathrm{mM}$ 2,2'-azino-bis(3-ethylbenzothiazoline-6-sulfonic acid) (ABTS) to its radical cation $\left(\varepsilon_{436}=29300 \mathrm{M}^{-1} \mathrm{~cm}^{-1}\right)$ in $0.1 \mathrm{M}$ sodium acetate $(\mathrm{pH} 5)$ at $24^{\circ} \mathrm{C}$.

For saccharification, an enzyme mixture of Cellic CTec2 and Cellic HTec2 (Novozymes, Bagsværd, Denmark) was used. Cellic CTec2 is a cellulase preparation that shows high $\beta$-glucosidase activity, while Cellic HTec 2 is a hemicellulase preparation with mainly endoxylanase activity. Overall cellulase activity was determined using filter paper (Whatman No. 1 filter paper strips; Whatman, Maidstone, UK) and $\beta$-glucosidase activity was measured using cellobiose as substrate on Cellic CTec2 cocktail (100 FPU/mL cellulase and 3,950 IU/mL $\beta$-glucosidase activity) [36]. Furthermore, xylanase activity was determined using birchwood xylan on Cellic HTec2 cocktail (1,300 IU/mL) [37].

One unit of enzyme activity was defined as the amount of enzyme that transforms $1 \mu \mathrm{mol}$ of substrate per minute.

\section{Microorganisms and media}

$S$. cerevisiae KE6-12 was used as the fermenting microorganism. This recombinant $S$. cerevisiae strain encoding xylose genes $(X R$ and $X D H)$ from $P$. stipitis and overexpressing the endogenous xylulokinase has been evolved to grow on lignocellulosic hydrolysates (Albers et al., unpublished results) [15]. Pre-inocula were grown for 24 hours at $30^{\circ} \mathrm{C}$ in $250 \mathrm{~mL}$ Erlenmeyer flasks shaken at $150 \mathrm{rpm}$ containing $50 \mathrm{~mL}$ of Delft medium as follows: $20 \mathrm{~g} / \mathrm{L}$ glucose, $20 \mathrm{~g} / \mathrm{L}$ xylose, $7.5 \mathrm{~g} / \mathrm{L}\left(\mathrm{NH}_{4}\right)_{2} \mathrm{SO}_{4}, 3.5 \mathrm{~g} / \mathrm{L} \mathrm{KH}_{2} \mathrm{PO}_{4}, 0.75 \mathrm{~g} / \mathrm{L}$ $\mathrm{MgSO}_{4} \cdot 7 \mathrm{H}_{2} \mathrm{O}, 2 \mathrm{~mL} / \mathrm{L}$ trace metal solution, and $1 \mathrm{~mL} / \mathrm{L}$ vitamin solution. The cells were harvested by centrifugation at 5,000 rpm for 5 minutes at room temperature. The supernatant was discarded and the pellet was washed once with sterile water. The cell pellet was then weighed and diluted with sterile water to obtain the desired inoculum size.

\section{Liquid fraction fermentation experiments}

Pretreated slurry $(26 \% \mathrm{DM} \mathrm{w} / \mathrm{v})$ was vacuum-filtered in order to obtain the liquid fraction. The recovered liquid fraction, that non-diluted is equivalent to $26 \% \mathrm{DM} \mathrm{w} / \mathrm{v}$, was further diluted to an equivalent DM concentration of
$14 \%$ and $12 \%(\mathrm{w} / \mathrm{v})$ with $0.05 \mathrm{M}$ citrate buffer, $\mathrm{pH} 5.5$. In addition, diluted liquid fractions were supplemented with diammonium phosphate (DAP; $5 \mathrm{~g} / \mathrm{L}$ ). Prior to fermentation, oligomers were enzymatically hydrolyzed at $50^{\circ} \mathrm{C}$ and $150 \mathrm{rpm}$ for 24 hours to obtain monomeric sugars with an enzyme loading of $0.5 \mathrm{FPU} / \mathrm{mL}$ Cellic CTec 2 and $2 \mathrm{IU} / \mathrm{mL}$ Cellic HTec2. These enzyme doses were selected according to previous optimization studies [38]. The temperature was then reduced to $35^{\circ} \mathrm{C}$, and liquid fractions equivalent to $14 \% \mathrm{DM}(\mathrm{w} / \mathrm{v})$ were inoculated with 1,3 , or $5 \mathrm{~g} / \mathrm{L}$ dry weight cell mass, while liquid diluted to $12 \% \mathrm{DM}(\mathrm{w} / \mathrm{v})$ was only fermented with an inoculum size of $5 \mathrm{~g} / \mathrm{L}$. Fermentation tests were performed in triplicate for 120 hours in $100 \mathrm{~mL}$ Erlenmeyer flasks shaken at $150 \mathrm{rpm}$, using rubber caps with a needle to allow outflow of $\mathrm{CO}_{2}$.

ANOVA was performed to identify differences in final yields between untreated and laccase-treated liquid fractions. ANOVA proves statistically whether the means of several groups are all different. The confidence level to identify statistically significant differences was $95.0 \%$.

\section{Simultaneous saccharification and co-fermentation (SSCF) processes}

SSCF experiments were run in two different operational modes: batch and fed-batch. For batch SSCF, pretreated slurry was diluted to $12 \%$ and $16 \%$ DM (w/v) consistencies with $0.05 \mathrm{M}$ citrate buffer, $\mathrm{pH}$ 5.5. Fed-batch cultures were performed at an initial substrate loading of $6 \% \mathrm{DM}(\mathrm{w} / \mathrm{v})$ and two pulses of substrate were added at 25 hours and 50 hours, reaching 12\% and 16\% DM (w/v), respectively, after each addition.

For saccharification, Cellic Ctec 2 at $15 \mathrm{FPU} / \mathrm{g}$ DM and Cellic HTec2 at $60 \mathrm{IU} / g$ DM were added according to previous optimization studies [38]. As nutrient, DAP at $5 \mathrm{~g} / \mathrm{L}$ was added. For batch SSCF, $1 \mathrm{~g} / \mathrm{L}$ S. cerevisiae KE6-12 was used for inoculation whereas $3 \mathrm{~g} / \mathrm{L}$ was used for fed-batch SSCF (taking into account the initial substrate loading, 6\% DM (w/v)). In the case of fed-batch SSCF, hydrolytic enzymes and DAP were added together with the substrate in order to keep the concentration of DAP at $5 \mathrm{~g} / \mathrm{L}$ and enzyme doses at $15 \mathrm{FPU} / \mathrm{g}$ DM substrate of Cellic CTec2 and 60 IU/g DM substrate of Cellic HTec2. Moreover, after each addition of substrate, the $\mathrm{pH}$ was adjusted to 5.5 with $10 \mathrm{M} \mathrm{NaOH}$.

All the experiments were run in triplicate at $35^{\circ} \mathrm{C}$ and 180 rpm for 144 hours (batch SSCF) or 168 hours (fed-batch SSCF). In the same way as in the fermentation tests, the SSCF processes were carried out in $100 \mathrm{~mL}$ shake flasks, using rubber caps with a needle to allow $\mathrm{CO}_{2}$ outflow.

\section{Laccase treatment}

Laccase was used to detoxify the liquid fraction before fermentation tests. For these experiments, P. cinnabarinus laccase at $1 \mathrm{IU} / \mathrm{mL}$ was added to diluted liquid 
fraction corresponding to $12 \%$ and $14 \%$ DM (w/v) after 21 hours of pre-hydrolysis (3 hours before inoculation).

In addition, laccase treatment was also carried out in SSCF experiments either in batch mode or fed-batch mode. When performing batch SSCF, $10 \mathrm{IU} / g$ substrate for laccase enzyme was added to the slurry at $12 \%$ or $16 \% \mathrm{DM}(\mathrm{w} / \mathrm{v})$ and incubated for 12 hours at $50^{\circ} \mathrm{C}$ and $180 \mathrm{rpm}$ before addition of hydrolytic enzymes and yeast. In the case of fed-batch assays, two different laccase addition strategies were studied. P. cinnabarinus laccase at $10 \mathrm{IU} / \mathrm{g}$ substrate (taking into account the final substrate loading) was added 21 hours after yeast inoculation to a substrate consistency of $6 \% \mathrm{DM}(\mathrm{w} / \mathrm{v})$ or 33 hours after inoculation, when the substrate concentration had reached $12 \% \mathrm{DM}(\mathrm{w} / \mathrm{v})$.

\section{Analytical methods}

Raw material and WIS fraction were analyzed using the standard National Renewable Energy Laboratory (NREL) methods for determination of structural carbohydrates and lignin in biomass [39]. Dry weight of slurry and WIS was determined by drying the samples at $105^{\circ} \mathrm{C}$ for 24 hours.

Total phenolic content of the supernatants was determined according to a slightly modified version of the Folin-Ciocalteu method [20].

Extracellular metabolites, sugars, 5-HMF, and furfural were analyzed by HPLC using an Aminex HPX-87H column with a $30 \times 4.6 \mathrm{~mm}$ Micro-Guard Cation-H column (Bio-Rad, Hercules, CA, USA) maintained at $45^{\circ} \mathrm{C}$. The eluent was $5 \mathrm{mM} \mathrm{H}_{2} \mathrm{SO}_{4}$ at a flow rate of $0.6 \mathrm{~mL} / \mathrm{min}$. Formic acid and acetic acid were determined under the same conditions, while maintaining the column at $65^{\circ} \mathrm{C}$.

Samples were taken at different times in the fermentation or SSCF process and centrifuged at 14,000 rpm for 3 minutes. Supernatant was filtered through $0.2 \mu \mathrm{m}$ nylon filters and stored at $-20^{\circ} \mathrm{C}$ until analysis.

Cell viability was determined as $\mathrm{CFU} / \mathrm{mL}$ by cell counting on agar plates $(20 \mathrm{~g} / \mathrm{L}$ glucose, $20 \mathrm{~g} / \mathrm{L}$ agar, 5 $\mathrm{g} / \mathrm{L}$ yeast extract, $2 \mathrm{~g} / \mathrm{L} \mathrm{NH} \mathrm{NH}_{4} \mathrm{Cl}, 1 \mathrm{~g} / \mathrm{L} \mathrm{KH}_{2} \mathrm{PO}_{4}$, and 0.3 $\left.\mathrm{g} / \mathrm{L} \mathrm{MgSO}_{4} \cdot 7 \mathrm{H}_{2} \mathrm{O}\right)$. The plates were incubated at $30^{\circ} \mathrm{C}$ for 48 hours before counting of colonies.

\footnotetext{
Abbreviations

5-HMF: 5-hydroxymethylfurfural; ABTS: 2,2'-azino-bis(3-ethylbenzothiazoline-6sulfonic acid); ANOVA: Analysis of variance; CFU: Colony-forming unit; DAP: Diammonium phosphate; DM: Dry matter; FPU: Filter paper unit; HPLC: High performance liquid chromatography; IU: International unit; NREL: National Renewable Energy Laboratory; SSCF: Simultaneous saccharification and co-fermentation; WIS: Water-insoluble solids; XDH: Xylitol dehydrogenase; XR: Xylose reductase.
}

\section{Competing interests}

LO does consultancy work for Taurus Energy AB, Lund, Sweden.

\section{Authors' contributions}

ADM, ETP, DI, MB, and LO participated in the design of the study. ADM and ETP performed the experimental work and wrote the manuscript. LO, DI, and
MB conceived the study and commented on the manuscript. All the authors read and approved the final manuscript.

\section{Acknowledgements}

Taurus Energy AB, Dr. Eva Albers, and Rakesh Koppram are gratefully acknowledged for providing the S. cerevisiae KE6-12 strain. The authors thank Dr. Ignacio Ballesteros for helping in the pretreatment. ADM acknowledges a CPI contract (Consejería de Educación-Comunidad de Madrid, Spain). ETP acknowledges the Swedish Energy Agency (Energimyndigheten) for financial support.

\section{Author details}

${ }^{1}$ Instituto IMDEA Energía, Biotechnological Processes for Energy Production Unit, Móstoles 28935, Spain. ${ }^{2}$ Department of Chemical and Biological Engineering, Industrial Biotechnology, Chalmers University of Technology, Göteborg SE-41296, Sweden. ${ }^{3}$ INIA-CIFOR, Forestry Products Department, Cellulose and Paper Laboratories, Madrid 28040, Spain. ${ }^{4}$ Centro de Investigaciones Energéticas, Medioambientales y Tecnológicas, Biofuels Unit, Madrid 28040, Spain

Received: 3 September 2013 Accepted: 29 October 2013

Published: 13 November 2013

\section{References}

1. Ballesteros M, Sáez F, Ballesteros I, Manzanares P, Negro MJ, Martínez JM, Castañeda R, Oliva Dominguez JM: Ethanol production from the organic fraction obtained after thermal pretreatment of municipal solid waste. Appl Biochem Biotechnol 2010, 161(1-8):423-431.

2. Alvira P, Tomás-Pejó E, Ballesteros M, Negro MJ: Pretreatment technologies for an efficient bioethanol production process based on enzymatic hydrolysis: a review. Bioresour Technol 2010, 101(13):4851-4861.

3. Tomás-Pejó E, Alvira P, Ballesteros M, Negro MJ: Pretreatment technologies for lignocellulose-to-bioethanol conversion. In Biofuels: Alternative Feedstocks and Conversion Processes. Edited by Pandey A, Larroche C, Ricke SC, Dussap CG, Gnansounou E. Burlington, MA: Elsevier; 2011:149-176.

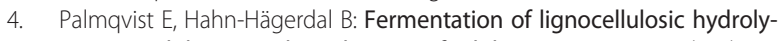
sates. II: inhibitors and mechanism of inhibition. Bioresour Technol 2000, 74:25-33.

5. Klinke HB, Thomsen AB, Ahring BK: Inhibition of ethanol-producing yeast and bacteria by degradation products produced during pre-treatment of biomass. Appl Microbiol Biotechnol 2004, 66(1):10-26.

6. Ximenes E, Kim Y, Mosier N, Dien B, Ladisch M: Inhibition of cellulases by phenols. Enzyme Microb Technol 2010, 46:170-176.

7. Ximenes E, Kim Y, Mosier N, Dien B, Ladisch M: Deactivation of cellulases by phenols. Enzyme Microb Technol 2011, 48:54-60.

8. Parawira W, Tekere M: Biotechnological strategies to overcome inhibitors in lignocellulose hydrolysates for ethanol production: review. Crit Rev Biotechnol 2011, 31(1):20-31.

9. Jurado M, Saparrat M, Martínez AT, Martínez MJ: Application of white-rot fungi in transformation, detoxification, or revalorization of agriculture wastes: role of laccase in the processes. Comprehen Biotechnol 2011, 6:595-603.

10. Jönsson JL, Palmqvist E, Nilvebrant NO, Hahn-Hägerdal B: Detoxification of wood hydrolysates with laccase and peroxidase from the white-rot fungus Trametes versicolor. Appl Microbiol Biotechnol 1998, 49:691-697.

11. Martín C, Galbe M, Wahlbom CF, Hahn-Hägerdal B, Jönsson JL: Ethanol production from enzymatic hydrolysates of sugarcane bagasse using recombinant xylose utilising Saccharomyces cerevisae. Enzyme Microbiol Technol 2002, 31:274-282.

12. Jurado M, Prieto A, Martínez-Alcalá A, Martínez AT, Martínez MJ: Laccase detoxification of steam-exploded wheat straw for second generation bioethanol. Bioresour Technol 2009, 100:6378-6384.

13. Kalyani D, Dhiman SS, Kim H, Jeya M, Kim I-W, Lee J-K: Characterization of a novel laccase from the isolated Coltricia perennis and its application to detoxification of biomass. Process Biochem 2012, 47:671-678.

14. Moreno AD, Ibarra D, Fernández JL, Ballesteros M: Different laccase detoxification strategies for ethanol production from lignocellulosic biomass by the thermotolerant yeast Kluyveromyces marxianus CECT 10875. Bioresour Technol 2012, 106:101-109.

15. Koppram R, Nielsen F, Albers E, Lambert A, Wännström S, Welin L, Zacchi G, Olsson L: Simultaneous saccharification and co-fermentation for 
bioethanol production using corncobs at lab, PDU and demo scales. Biotechnol Biofuels 2013, 6(1):2.

16. Almeida JRM, Modig T, Petersson A, Hahn-Hägerdal B, Liden G, GorwaGrauslund MF: Increased tolerance and conversion of inhibitors in lignocellulose hydrolysates by Saccharomyces cerevisiae. J Chem Technol Biotechnol 2007, 82:340-349.

17. Taherzadeh MJ, Karimi K: Fermentation inhibitors in ethanol processes and different strategies to reduce their effects. In Biofuels: Alternatives Feedstocks and Conversion Processes. Edited by Pandey A, Larroche C, Ricke SC, Dussap CG, Gnansounou E. Burlington, MA: Elsevier; 2011:287-311.

18. Oliva JM, Sáez F, Ballesteros I, González A, Negro MJ, Manzanares P, Ballesteros M: Effect of lignocellulosic degradation compounds from steam explosion pretreatment on ethanol fermentation by thermotolerant yeast Kluyveromyces marxianus. Appl Biochem Biotechnol 2003, 105:141-154

19. Tomás-Pejó E, Oliva JM, González A, Ballesteros I, Ballesteros M: Bioethanol production from wheat straw by the thermotolerant yeast Kluyveromyces marxianus CECT 10875 in a simultaneous saccharification and fermentation fed-batch process. Fuel 2009, 88:2142-2147.

20. Alvira P, Moreno AD, Ibarra D, Sáez F, Ballesteros M: Improving the fermentation performance of Saccharomyces cerevisiae by laccases during ethanol production from steam-exploded wheat straw at high substrate loadings. Biotechnol Prog 2013, 29(1):74-82.

21. Moreno AD, Ibarra D, Ballesteros I, González A, Ballesteros M: Comparing cell viability and ethanol fermentation of the thermotolerant yeast Kluyveromyces marxianus and Saccharomyces cerevisiae on steamexploded biomass treated with laccase. Bioresour Technol 2013, 135:239-245.

22. Kolb M, Sieber V, Amann M, Faulstich M, Schieder M: Removal of monomer delignification products by laccase from Trametes versicolor. Bioresour Technol 2012, 104:298-304

23. Rosgaard L, Andric P, Dam-Johansen K, Pedersen S, Meyer AS: Effects of substrate loading on enzymatic hydrolysis and viscosity of pretreated barley straw. Appl Biochem Biotechnol 2007, 143:27-40.

24. Moreno AD, Tomás-Pejó E, Ibarra D, Ballesteros M, Olsson L: In situ laccase treatment enhances the fermentability of steam-exploded wheat straw in SSCF processes at high dry matter consistencies. Bioresour Technol 2013, 143:337-343.

25. Meinander NQ, Boels I, Hahn-Hägerdal B: Fermentation of xylose/glucose mixtures by metabolically engineered Saccharomyces cerevisiae strains expressing XYL1 and XYL2 from Pichia stipitis with and without overexpression of TAL1. Bioresour Technol 1999, 68:79-87.

26. Alvira P, Negro MJ, Ballesteros M: Effect of endoxylanase and a-Larabinofuranosidase supplementation on the enzymatic hydrolysis of steam exploded wheat straw. Bioresour Technol 2011, 102:4552-4558.

27. Horn SJ, Vaaje-Kolstad G, Westereng B, Eijsink VGH: Novel enzymes for the degradation of cellulose. Biotechnol Biofuels 2012, 5:45.

28. Abdel-Banat BM, Hoshida H, Ano A, Nonklang S, Akada R: Hightemperature fermentation: how can processes for ethanol production at high temperatures become superior to the traditional process using mesophilic yeast? Appl Microbiol Biotechnol 2010, 85(4):861-867.

29. Zacchi G, Axelsson A: Economic-evaluation of preconcentration in production of ethanol from dilute sugar solutions. Biotechnol Bioeng 1989, 34:223-233

30. Banerjee S, Mudliar S, Sen R, Giri B, Staputi D, Chakrrabarti T, Pandey RA: Commercializing lignocellulosic bioethanol: technology bottlenecks and possible remedies. Biofuels Bioprod Biorefin 2009, 4:77-93.

31. Olofsson K, Rudolf A, Liden G: Designing simultaneous saccharification and fermentation for improved xylose conversion by a recombinant strain of Saccharomyces cerevisiae. J Biotechnol 2008, 134:112-120.

32. Tomás-Pejó E, Ballesteros M, Oliva JM, Olsson L: Adaptation of the xylose fermenting yeast Saccharomyces cerevisiae F12 for improving ethanol production in different fed-batch SSF processes. I Ind Microbiol Biotechnol 2010, 37(11):1211-1220.

33. Jørgensen $\mathrm{H}$, Kristensen JB, Felby C: Enzymatic conversion of lignocellulose into fermentable sugars: challenges and opportunities. Biofuels Bioprod Bioref 2007, 1:119-134.

34. Kristensen JB, Felby C, Jørgensen H: Yield-determining factors in high-solids enzymatic hydrolysis of lignocellulose. Biotechnol Biofuels 2009, 2:11.
35. Wang W, Kang L, Wei H, Arora R, Lee YY: Study on the decreased sugar yield in enzymatic hydrolysis of cellulosic substrate at high solid loading. Appl Biochem Biotechnol 2011, 164:1139-1149.

36. Ghose TK: Measurement of cellulase activity. Pure App/ Chem 1987, 59:257-268

37. Bailey MJ, Biely P, Poutanen $\mathrm{K}$ : Interlaboratory testing of methods for assay xylanase activity. J Biotechnol 1991, 23:257-270.

38. Barta Z, Duque A, Oliva JM, Manzanares P, González A, Ballesteros M, Réczey K: Improving Enzymatic Hydrolysis of Alkali-Extruded Barley Straw by Varying the Dosage and Ration of Hemicellulases and Cellulases, Italic 6 Science and Technology of Biomass: Advances and Challenges. Viterbo: Tuscia University; 2011

39. National Renewable Energy Laboratory (NREL): Standard Biomass Analytical Procedures. Golden, CO: NREL; 2011. http://www.nrel.gov/biomass/ analytical_procedures.html.

doi:10.1186/1754-6834-6-160

Cite this article as: Moreno et al:: Fed-batch SSCF using steam-exploded wheat straw at high dry matter consistencies and a xylose-fermenting Saccharomyces cerevisiae strain: effect of laccase supplementation. Biotechnology for Biofuels 2013 6:160

\section{Submit your next manuscript to BioMed Central and take full advantage of:}

- Convenient online submission

- Thorough peer review

- No space constraints or color figure charges

- Immediate publication on acceptance

- Inclusion in PubMed, CAS, Scopus and Google Scholar

- Research which is freely available for redistribution 